\title{
Effects of a Common Variant in the CD38 Gene on Social Processing in an Oxytocin Challenge Study: Possible Links to Autism
}

\author{
Carina Sauer*,', Christian Montag ${ }^{2}$, Christiane Wörner', Peter Kirsch' ${ }^{1,3}$ and Martin Reuter ${ }^{2,3}$ \\ 'Department of Clinical Psychology, Central Institute of Mental Health, Medical Faculty Mannheim, Heidelberg University, Mannheim, Germany; \\ ${ }^{2}$ Department of Differential and Biological Psychology, Rheinische Friedrich-Wilhelms-University, Bonn, Germany
}

\begin{abstract}
The intranasal application of oxytocin (OT) has been shown to influence behavioral and neural correlates of social processing. These effects are probably mediated by genetic variations within the OT system. One potential candidate could be the CD38 gene, which codes for a transmembrane protein engaged in OT secretion processes. A common variation in this gene (rs3796863) was recently found to be associated with autism spectrum disorders (ASD). Using an imaging genetics approach, we studied differential effects of an intranasal OT application on neural processing of social stimuli in 55 healthy young men depending on their CD38 gene variant in a double-blind placebo-controlled crossover design. Genotype had a significant influence on both behavioral and neuronal measures of social processing. Homozygotic risk allele carriers showed slower reaction times (RT) and higher activation of left fusiform gyrus during visual processing of social stimuli. Under OT activation differences between genotypes were more evident (though not statistically significantly increased) and RT were accelerated in homozygotic risk allele carriers. According to our data, rs 3796863 mainly influences fusiform gyrus activation, an area which has been widely discussed in ASD research. OT seems to modulate this effect by enhancing activation differences between allele groups, which suggests an interaction between genetic makeup and OT availability on fusiform gyrus activation. These results support recent approaches to apply OT as a pharmacological treatment of ASD symptoms.

Neuropsychopharmacology (2012) 37, I474-|482; doi:I0.1038/npp.201।.333; published online 25 January 2012
\end{abstract}

Keywords: oxytocin; CD38; social processing; imaging genetics; autism

\section{INTRODUCTION}

The neuropeptide oxytocin (OT) has been implicated in a broad range of social processes and behavior (MeyerLindenberg et al, 2011). Studies on healthy humans eg revealed that OT improves mind reading (Domes et al, 2007b), increases fixation of the eye region (Guastella et al, 2008), promotes trust (Kosfeld et al, 2005), in-group favoritism (De Dreu et al, 2010, 2011), and positive communication during couple conflicts (Ditzen et al, 2009). It also has diminishing effects on negative social emotions like social stress or anxiety (Ditzen et al, 2009; Guastella et al, 2009; Heinrichs et al, 2003). Altogether OT

*Correspondence: C Sauer, Department of Clinical Psychology, Central Institute of Mental Health, Medical Faculty Mannheim, Heidelberg University, J5, Mannheim 68159, Germany, Tel: +49 621 I7036154, Fax: + 49621 17036505,

E-mail: Carina.Sauer@zi-mannheim.de

${ }^{3}$ These authors contributed equally to this work.

Received 30 August 201 I; revised 20 December 201 I; accepted 20 December 2011 seems to serve the continued propagation of the species (Lee et al, 2009).

Additionally, OT has been implicated in states of aberrant social functioning like in autism spectrum disorders (ASD). Indeed, first attempts to improve social deficits in ASD via OT administration revealed positive results: improved retention of affective speech comprehension (Hollander et al, 2007), enhanced mind-reading performance (Guastella et al, 2010), more frequent engagement in positive social interactions, and enhanced feelings of trust and preference towards partners within positive interactions (Andari et al, 2010).

On a neuronal level, the amygdala seems to have an important role in mediating the effects of OT on social and emotional processing. The amygdala contains a high density of OT receptors (Huber et al, 2005); moreover several functional magnetic resonance imaging (fMRI) studies revealed OT induced decreases of amygdala activation during social or fear processing (Baumgartner et al, 2008; Domes et al, 2007a; Kirsch et al, 2005; Petrovic et al, 2008). Although most fMRI studies on OT published so far focus on the amygdala, there are other potentially interesting 
brain areas like the fusiform gyrus which are also involved in processing of social stimuli. The fusiform gyrus is highly relevant for face perception (Haxby et al, 2000, 2002; Kanwisher and Yovel, 2006) and is directly connected to the amygdala via reciprocal projections (Freese and Amaral, 2005, 2006). Interestingly, one OT challenge study also revealed OT induced decreases in activation of the fusiform gyrus (Petrovic et al, 2008).

As complex neuropsychiatric disorders like ASD as well as many circumscribed psychological functions are highly heritable, approaches that try to bridge the gap between genetics and observable phenotypes have become very popular. One promising attempt is the investigation of 'intermediate phenotypes' via imaging genetics studies (Meyer-Lindenberg and Weinberger, 2006). Several studies revealed associations between ASD and different single nucleotide polymorphisms (SNPs) on the OT receptor gene (Gregory et al, 2009; Jacob et al, 2007; Lerer et al, 2008; Wermter et al, 2010; Wu et al, 2005; Yrigollen et al, 2008). However, the relevance and mechanisms of particular SNPs for the ASD phenotype remain largely unclear. The same is true for the influence of these common variants on brain activation, structure or connectivity in healthy humans. Although evidence has been provided for an intermediate phenotype related to one of these receptor gene variations (rs53576) (Tost et al, 2010), so far there is no study that has concentrated on other aspects of the OT system like OT production, storage, or secretion. Recently, the transmembrane protein $\mathrm{CD} 38$ has received particular attention as it is engaged in OT secretion in the central nervous system and influences social behavior. $C D 38^{-1-}$ knockout mice show deficits in social memory processes and maternal nurturing behavior (Higashida et al, 2010; Jin et al, 2007). Moreover, two recent studies revealed an association between a common variation in the $C D 38$ gene and ASD in a sample of 252 white US families (Munesue et al, 2010) and in a sample of 149 Israeli Jewish families (Lerer et al, 2010): rs3796863 with the C-allele as the risk allele. Additionally, the C-allele was found to be associated with lower CD38 expression in lymphoblastoid cells (Lerer et al, 2010).

In the present study we addressed the question if this genetic variant affects brain activation and behavior during basal social perception processes. Furthermore, we extended the imaging genetics approach by investigating how the effect of a pharmacological challenge with OT is influenced by this CD38 gene variant. This procedure enabled us to detect potential interactions between OT availability and dispositions. As ASD studies have mainly reported hypoactivation of amygdala and fusiform areas in patients (Jemel et al, 2006; Schultz, 2005; Verhoeven et al, 2010), we expected reduced activation in both structures in homozygotic carriers of the autism-associated C-allele during processing of social stimuli. Under OT we expected a more pronounced effect in homozygotic $\mathrm{C}$-allele carriers because they potentially suffer from a less effective OT system.

\section{MATERIALS AND METHODS}

\section{Participants}

We investigated 56 healthy young men with no history of psychiatric or neurological illness. All were non-smokers or
Table I Sample Characteristics

\begin{tabular}{lccc}
\hline rs3796863 & CC genotype & CA genotype & AA genotype \\
\hline$N$ & 30 & 23 & 2 \\
\cline { 3 - 4 } & & 25 & \\
& Mean \pm SD & Mean \pm SD & P \\
\hline Age (years) & $25.2 \pm 2.76$ & $24.6 \pm 2.42$ & 0.40 \\
Education (years) & I5.0 \pm 0.67 & I5.I \pm 0.60 & 0.62 \\
Substance order & OT first: 13 & OT first: |4 & 0.35 \\
& PLA first: 17 & PLA first: I I &
\end{tabular}

Abbreviations: OT, oxytocin; PLA, placebo.

smoked only occasionally and, except for one, were righthanded. One participant was excluded after completion of the study because he reported afterwards that he had donated plasma in the morning of the first session. Accordingly, the final sample consisted of 55 males; 30 subjects were homozygotic carriers of the risk allele (CC) with respect to $r s 3796863,23$ were heterozygotes (CA), and 2 were homozygotic carriers of the protective allele (AA). According to the PubMed database, $\mathrm{CC}$ and $\mathrm{AC}$ are common genetic variants occurring in $\sim 50$ and $46 \%$ of the Caucasian population, whereas only $4 \%$ are AA carriers (http://www.ncbi.nlm.nih.gov/projects/SNP). For statistical analyses, we compared a combined group of $\mathrm{CA}$ and $\mathrm{AA}$ genotypes $(\mathrm{A}+$, the 'lower risk group') with $\mathrm{CC}$ carriers (A-, the 'higher risk group'). Groups did not differ in age, education, or order of substance application, although group assignment was performed post hoc according to genotype (Table 1).

The study was approved by the ethics committee of the German Psychological Society (DGPs) and all participants gave written informed consent.

\section{Experimental Design and Procedure}

fMRI data were acquired in a double-blind placebocontrolled crossover design. Participants attended two sessions scheduled at the same time of day with an interval of 1 week. For each session, they were asked to abstain from alcohol and cigarettes for $12 \mathrm{~h}$ before the experiment and from caffeine for $3 \mathrm{~h}$.

In the first session, participants were first asked to complete a screening questionnaire concerning actual somatic illnesses and the Symptom Checklist 90-R (Franke, 2002) for a broad range of psychological problems. Afterwards, in each session participants received either placebo (PLA) or a dose of 25 IU of OT (Syntocinon Spray, Novartis, Austria) via intranasal application around $30 \mathrm{~min}$ before the start of the first fMRI experiment. Participants administered the substance themselves under observation of the investigator. In total, they administered five puffs alternating nostrils with each puff containing 5 IU. To control for substance effects on mood, arousal dominance, anxiety, anger, and wakefulness, participants completed the following questionnaires before substance administration and after the fMRI session: the Self Assessment Maniquin (SAM) 
(Bradley and Lang, 1994), the Multidimensional Mood State Questionnaire (MDBF) (Steyer et al, 1997), and the state components of the State-Trait Anger Expression Inventory (Schwenkmezger et al, 1992), and the State-Trait Anxiety Inventory (Laux et al, 1981).

Before the fMRI session, a 5-min structural MRI scan was performed to obtain anatomical data. Subsequently, we applied two different fMRI tasks, each implying functions of social cognition, which are highly relevant for ASD as well as for OT research: (1) processing of social emotional stimuli and (2) gaze processing (see Supplementary Figure S1 for experimental procedure and example trials of both tasks). The first paradigm was an extended version of the Hariri face matching task (Hariri et al, 2002b), which we also used in our previous study (Kirsch et al, 2005) and which has been consistently shown to elicit activation of amygdala as well as fusiform areas (Hariri et al, 2002a, b, 2003). Participants are required to match one of two simultaneously presented pictures with an identical target. The extended version consisted of blocks showing geometric shapes (control condition) or pictures of faces or scenes with either positive or negative valence (social emotional conditions). Facial stimuli for the matching paradigm were taken from the Ekman and Friesen series (1976) and consisted of angry/fearful faces (negative faces) or happy faces (positive faces). Pictures of social scenes were taken from the International Affective Picture System (Lang et al, 2008). Negative scenes depicted fear-eliciting social interactions, eg one person threatening another one with a weapon, whereas positive scenes depicted couples in an erotic interaction. Each control task block lasted for $15 \mathrm{~s}$ and consisted of six matching trials à $2.5 \mathrm{~s}$, whereas each social-emotional task block had a duration of $30 \mathrm{~s}$ with six matching trials à $5 \mathrm{~s}$. There was no interval between the offset of one trial and the onset of the next one, and the stimulus duration was independent of the participant's response. The participant's response was recorded during the trial duration of $5 \mathrm{~s}$. Altogether, every social-emotional task block (negative scenes, positive scenes, negative faces, and positive faces) was presented four times in a nonrandomized order and was followed by a control task block each time. Accordingly, the total duration of the matching paradigm was about 13 min.

The second paradigm on gaze processing was used because direct eye gaze is often avoided by ASD patients and the amygdala has been implicated in the processing of gaze direction (Itier and Batty, 2009; Kawashima et al, 1999). It was applied $\sim 44 \mathrm{~min}$ after OT application and was realized within an event-related design. One trial consisted of a picture of a person with either direct or averted eye gaze and participants were asked to indicate via button press whether the person looked to the left, to the right, or directly to the participant. Stimulus presentation was randomized and lasted for $3 \mathrm{~s}$, independent of the participant's response, with a mean interstimulus interval (offset of a stimulus to onset of the next one) of $5 \mathrm{~s}$. During the interstimulus interval a fixation cross was presented. The participant's response was recorded during stimulus presentation and adjacent interstimulus interval until the onset of the next stimulus. In total, the second experiment consisted of 64 trials, 32 with direct eye gaze and 32 with averted eye gaze (16 left and 16 right). Persons shown on the photos were of mixed gender (eight male and eight female) and age ( $20-60$ years).

\section{fMRI Data Acquisition}

Scanning was performed on a $3 \mathrm{~T}$ Siemens TRIO scanner with the following parameters for the functional MRI scans using EPI sequences: 30 axial slices à $4 \mathrm{~mm}, 1 \mathrm{~mm}$ gap, $\mathrm{TR}=2 \mathrm{~s}, \mathrm{TE}=30 \mathrm{~ms}, \mathrm{FoV} 192 \times 192 \mathrm{~mm}$, and flip angle $80^{\circ}$. Anatomical data were obtained from a T1-weighted threedimensional MPRAGE sequence (192 sagittal slices of $1 \mathrm{~mm}$ thickness, $\mathrm{TR}=2.3 \mathrm{~s}, \mathrm{TE}=3.03 \mathrm{~ms}$, FoV $256 \times 256 \mathrm{~mm}$, and flip angle $9^{\circ}$ ).

\section{fMRI Data Analyses}

fMRI data analysis was conducted by means of SPM8 (http:// www.fil.ion.ucl.ac.uk/spm). Preprocessing procedures were identical for both paradigms. Images were realigned to the first image, slice-time corrected, spatially normalized into a standard stereotactic space with a voxel size of $2 \times 2 \times 2 \mathrm{~mm}$ using the Montreal Neurological Institute template and smoothed with an $8 \mathrm{~mm}$ full width at half maximum Gaussian kernel.

On the first level, a general linear model (GLM) incorporating both substance conditions (PLA, and OT) as separate sessions was applied for each paradigm. For each session, task regressors as well as regressors for the six motion parameters from realignment were included in the model, five task regressors for the matching task (one for each experimental condition: forms, negative faces, positive faces, negative scenes, and positive scenes) and two task regressors for the gaze processing task (direct gaze and averted gaze). First level contrasts were defined for both substance conditions combined (OT + PLA) as well as separately (OT and PLA) and for the comparison between substances (OT vs PLA).

To enable us to investigate general aspects of social $v s$ non-social processing as well as more specific aspects of social processing we also specified different kinds of contrasts depending on task condition: for the matching task, we defined one contrast comparing all social conditions to the control condition ('social > non-social') as well as each social condition separately to the control condition ('negative faces $>$ non-social', 'positive faces $>$ non-social', 'negative scenes $>$ non-social', and 'positive scenes $>$ nonsocial'). Contrasts for the gaze processing task were defined as 'direct + averted $>$ low-level baseline' and 'averted $>$ direct' and 'direct $>$ averted'. Finally, when combining these task conditions with the substance conditions specified above, we eg defined the following first level contrasts for the investigation of general social processing in the first paradigm: 'social > non-social' (PLA), 'social > non-social' (OT), 'social > non-social' (OT + PLA), and 'social > non-social' (OT vs PLA).

In subsequent second level analyses, the two genotypes were compared with two sample $t$-tests (A- (CC genotype) $v s \mathrm{~A}+$ allele (genotypes $\mathrm{AA}$ and $\mathrm{AC}$ )) based on the contrasts resulting from first level analyses. Additionally, substance order was entered as a covariate of no interest to control for potential influences, because group assignment was performed post hoc based on participants' genotype (though order did not differ between genotype groups). For results presented in the paper, significance threshold was set to $p<0.05$ corrected for multiple comparisons using Family 
Wise Error (FWE) correction for the whole brain analyses as well as for Region of Interest (ROI) analyses conducted for the amygdala and the fusiform gyrus. Both ROIs were anatomically defined (Anatomical Automatic Labeling) masks provided by the Wake Forest University PickAtlas software (http://fmri.wfubmc.edu). This software implemented in SPM 8 runs ROI analyses voxel by voxel and corrects for the number of voxels within the particular mask. For further exploration of the data we also performed the whole brain analyses at a more liberal significance threshold of $p<0.001$ uncorrected. Results from these analyses can be found in the Supplementary Information (Supplementary Table S1). For all analyses an additional cluster size threshold was set to a minimum of $k=10$ contiguously activated voxels.

\section{Behavioral Data Analyses}

Behavioral data in terms of reaction times (RT) and correct responses were analyzed using GLM $2 \times 2 \times 2$ repeated measures procedures implemented in PASW Statistics 18 (SPSS, Chicago, IL). Models consisted of task (matching task: social $v s$ non-social; gaze processing task: averted $v s$ direct) and substance conditions (OT vs PLA) as withinsubject factors, allele $(\mathrm{A}-v s \mathrm{~A}+)$ as between-subject factor and substance order as a covariate of no interest. For analyses of RT we used the individual person's median rather than the mean because the median is much more robust against outliers. Subsequently, within the GLM, mean values were calculated across individuals' median RT.

Self-reported mood, arousal and so on were analyzed in a similar way by means of $2 \times 2$ repeated measure analyses of variance, consisting of difference scores (pre vs post substance application) for each substance condition as within subject factor, genotype as between subject factor, and order of substance application as a covariate of no interest.

\section{Genotyping Procedures}

DNA was extracted from the buccal cells to avoid a selective exclusion of subjects with blood and injection phobia. Genotyping of the CD38 SNP ( $r s 3796863$ ) was performed by real-time polymerase chain reaction using a fluorescence melting curve detection analysis (for details see Supplementary Materials and Methods).

\section{RESULTS}

\section{Influence of OT and Genotype on Subjective Ratings of Mood, Arousal and so on}

Analyses of subjective ratings revealed only two significant results. Firstly, comparing differences between participants' subjective ratings pre and post substance application for both substance conditions resulted in a significant main effect of substance condition for the mood scale of the SAM (Bradley and Lang, 1994): $\mathrm{F}(1,50)=4.144, p=0.047$. Although participants reported a slightly reduced positive mood under PLA, the opposite was true for the OT condition where they reported a minimal increase in positive mood.

Secondly, we found an interaction between genotype and substance for the wakefulness scale of the MDBF (Steyer et al, 1997): $\mathrm{F}(1,51)=8.845, p=0.004$. Although there was a general increase of fatigue for the whole sample, A+ carriers reported a smaller increase of fatigue from pre to post application under OT compared with PLA. No such effect could be detected in A- carriers.

For none of the other measures a significant main effect of substance or genotype occurred. After applying correction for multiple comparisons (altogether eight individual models (one for each rating scale), therefore $\alpha_{\text {corr }}=0.05$ / $8=0.00625$ ), only the second result survived.

\section{Task Performance: Median RT and Accuracy}

Matching task. Analyses of median RTs in the matching task revealed a significant main effect of task condition $(\mathrm{F}(1,52)=165.3, p<0.001)$. Participants responded faster to non-social forms than to social stimuli. Genotype groups differed significantly regarding RTs (main effect of genotype: $\mathrm{F}(1,52)=4.4, p=0.041$ ) with $\mathrm{A}$ - carriers showing slower RT than $\mathrm{A}+$.

Moreover, we found interaction effects between factors. Firstly, there was a trend for an interaction between task condition and genotype $(\mathrm{F}(1,52)=3.202, p=0.079)$. Post
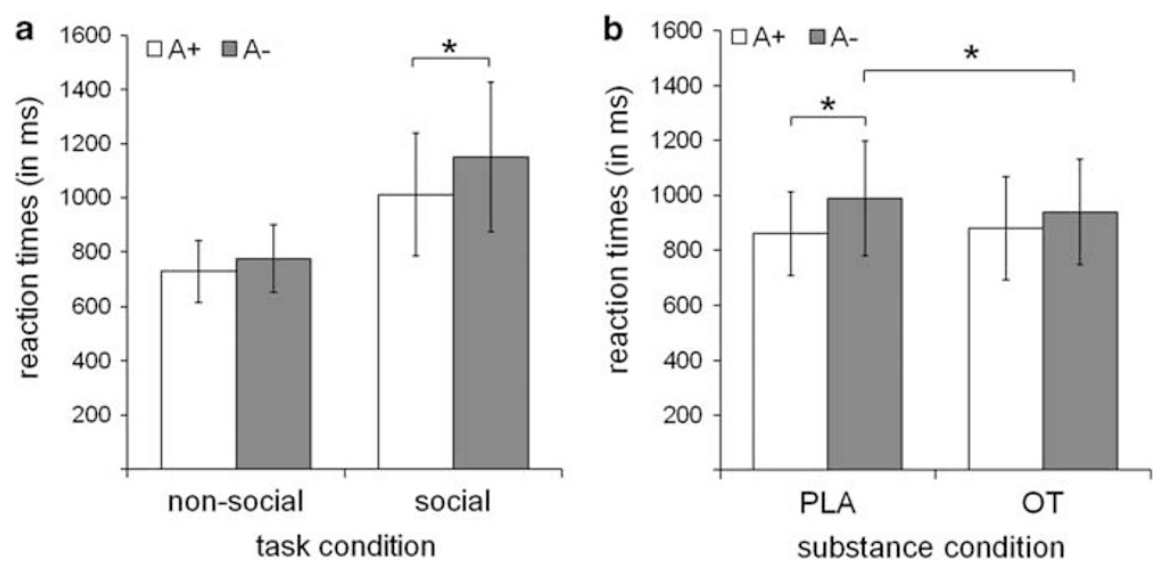

Figure I Median RT and SD in the matching paradigm. (a) Interaction effect of task condition and genotype for both substance conditions combined. (b) Interaction effect of substance condition and genotype for both task conditions combined. *Indicates significant differences according to post hoc tests $(p<0.05)$. 
hoc tests indicate a difference between allele groups only for social $(p=0.040)$ but not for non-social stimuli $(p=0.121)$ with A- carriers being slower than A+ (Figure 1a). Secondly, we found another trend for an interaction between substance condition and $\mathrm{A}-$ allele $(\mathrm{F}(1,52)=$ $3.979, p=0.051)$. According to post hoc tests OT led to a reduction of RT in $\mathrm{A}-$ carriers $(p=0.032)$, but not in $\mathrm{A}+$ $(p=0.489)$. Moreover, genotype groups differed under PLA $(p=0.010)$ but not under OT $(p=0.190)$ (Figure 1b).

Regarding accuracy in terms of percent correct responses, we could only find a main effect of task condition with a better performance for social stimuli than for non-social forms $(\mathrm{F}(1,52)=23.583, p<0.001)$. Moreover, there was a trend for an interaction between task condition and substance order $(\mathrm{F}(1,52)=4.039, p=0.050)$. Participants who received OT in the second session showed a bigger difference between the number of correct responses in both task conditions $(p<0.001)$ than those participants who received OT in the first session $(p=0.051)$. But as the task was very easy, we could observe a strong ceiling effect (Table 2).

Gaze processing task. With respect to the gaze processing task, neither analyses of RTs nor of percent correct responses yielded significant results (for details on RT and accuracy see Table 2).

\section{Brain Activation}

\section{General Processing of Social vs Non-Social Stimuli.}

Matching task (contrast 'social>non-social'): In general, processing of social stimuli compared with non-social forms led to a widespread activation of visual and limbic brain regions, among these amygdala and fusiform gyrus bilaterally ( $p<0.05$ FWE-corrected).

Comparison of allele groups $(\mathrm{A}->\mathrm{A}+)$ for processing of social stimuli compared with non-social forms during both substance conditions combined (OT + PLA) resulted in one single cluster located in the left fusiform gyrus, which was more highly activated in A- carriers $(k=42,(-20,-32$, $-18), t=5.62, p=0.012$ FWE-corrected) (Figure 2).

Investigation of both substance conditions separately showed that the fusiform cluster was more highly activated in A- mainly under OT $(k=57,(-30,-40,-20), t=6.21$, $p=0.002$ FWE-corrected) but not under PLA (Figure 3). However, the difference contrast (OT > PLA) did not reveal significant differences between substance conditions at a significance threshold of $p<0.05$ FWE-corrected, neither in the whole brain nor in ROI analyses using a fusiform gyrus mask.

The reversed allelic contrast $(\mathrm{A}+>\mathrm{A}-)$ did not produce significant results for substance conditions combined, for separate conditions, or for the comparison of conditions. ROI analyses of amygdala activation did not reveal significant results either.

Gaze processing task (contrast 'direct + averted $>$ lowlevel baseline'): Processing of facial stimuli compared with a low level baseline in the gaze processing task led to a widespread activation of visual and other face-processing areas, which included strong activation of fusiform gyri
Table 2 Reaction Times (RT, Median \pm SD) and Accuracy (mean percent correct responses $\pm \mathrm{SD}$ ) for (A) the matching task and (B) the gaze processing task for both substance conditions

\begin{tabular}{|c|c|c|}
\hline & $A-(C C)$ & $A+(A C, A A)$ \\
\hline \multicolumn{3}{|c|}{ (A) Matching task } \\
\hline & Median \pm SD (ms) & Median $\pm S D$ (ms) \\
\hline \multicolumn{3}{|c|}{ RT social } \\
\hline PLA & | $182.06 \pm 298.16$ & $995.98 \pm 201.94$ \\
\hline OT & | | | $9.92 \pm 255.87$ & $1027.93 \pm 252.12$ \\
\hline \multicolumn{3}{|c|}{ RT forms } \\
\hline PLA & $793.18 \pm 121.41$ & $723.90 \pm 103.05$ \\
\hline \multirow[t]{2}{*}{ OT } & $757.92 \pm 128.15$ & $731.12 \pm 122.73$ \\
\hline & Mean \pm SD (\%) & Mean $\pm S D(\%)$ \\
\hline \multicolumn{3}{|c|}{ Accuracy social } \\
\hline PLA & $99.34 \pm 1.08$ & $99.33 \pm 1.04$ \\
\hline OT & $99.10 \pm 1.61$ & $98.37 \pm 2.33$ \\
\hline \multicolumn{3}{|c|}{ Accuracy forms } \\
\hline PLA & $97.81 \pm 2.34$ & $97.83 \pm 2.44$ \\
\hline OT & $97.88 \pm 3.62$ & $97.34 \pm 3.38$ \\
\hline \multicolumn{3}{|c|}{ (B) Gaze processing task } \\
\hline & Median $\pm S D$ (ms) & Median $\pm S D$ (ms) \\
\hline \multicolumn{3}{|c|}{ RT direct } \\
\hline PLA & $948.45 \pm 183.67$ & $885.61 \pm 157.50$ \\
\hline OT & $941.85 \pm 230.03$ & $890.88 \pm 215.24$ \\
\hline \multicolumn{3}{|c|}{ RT averted } \\
\hline PLA & $969.01 \pm 197.22$ & $878.25 \pm 186.60$ \\
\hline \multirow[t]{2}{*}{ ОТ } & $952.91 \pm 223.01$ & $863.73 \pm 164.74$ \\
\hline & Mean \pm SD (\%) & Mean \pm SD (\%) \\
\hline \multicolumn{3}{|c|}{ Accuracy direct } \\
\hline PLA & $97.61 \pm 5.49$ & $98.88 \pm 2.69$ \\
\hline OT & $97.29 \pm 8.03$ & $98.63 \pm 2.03$ \\
\hline \multicolumn{3}{|c|}{ Accuracy averted } \\
\hline PLA & $98.75 \pm 3.13$ & $98.00 \pm 5.17$ \\
\hline OT & $97.40 \pm 9.32$ & $98.63 \pm 3.00$ \\
\hline
\end{tabular}

Abbreviations: OT, oxytocin; PLA, placebo.

and moderate activation of more lateral parts of the amygdala.

To confirm our results of higher fusiform activation in Acarriers in a second data set, we performed ROI analyses for the fusiform gyri in the gaze processing task. We found a significantly increased left fusiform activation for A - carriers compared with $\mathrm{A}+$ during processing of facial stimuli in both substance conditions combined (OT + PLA) in a cluster very similar to that of the matching task $(k=71,(-28,-42$, -16 ), $t=4.78, p=0.003$ FWE-corrected for the ROI (see Supplementary Figure S2 for illustration of the overlap). Analyzing both substance conditions separately led to a higher activation for $\mathrm{A}-$ carriers compared with $\mathrm{A}+$ under OT $(k=27,(-30,-44,-16), t=4.26, p=0.015$ FWEcorrected for the ROI) but not under PLA. Again, the 


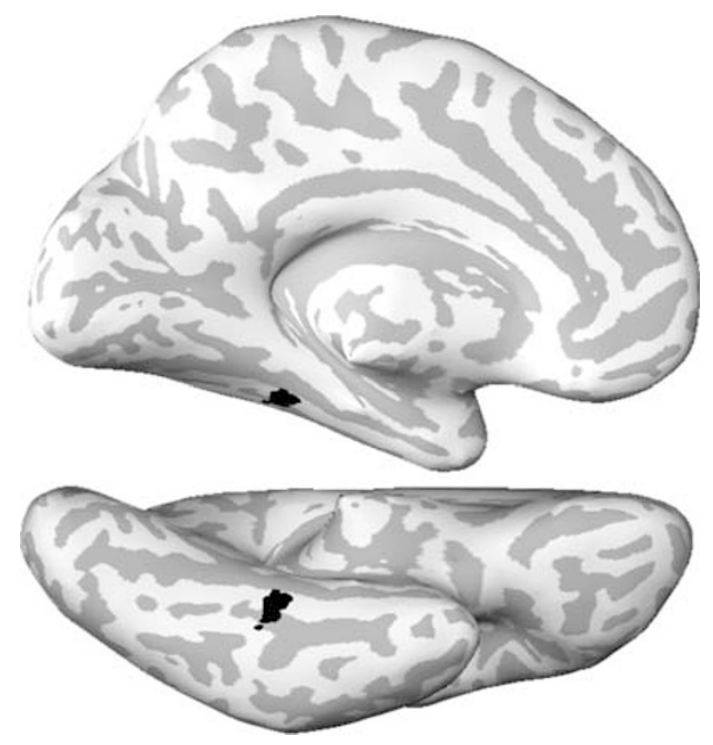

Figure 2 Voxels of the fusiform gyrus with increased activation in the A - group for the contrast 'social > non-social' (both substance conditions combined) superimposed on an inflated standard brain. Display thresholded at $p<0.05$ FWE-corrected for the whole brain. Note that the image was not masked and there were no voxels outside the fusiform gyrus found significantly activated.

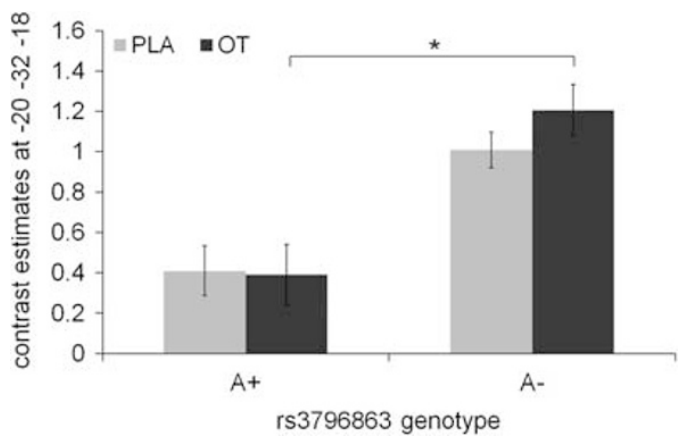

Figure 3 Mean contrast estimates \pm SEM for the left fusiform gyrus peak voxel of the contrast 'social>non-social' (both substance conditions combined) in the matching paradigm. *Indicates significant differences $(p<0.05$ FWE-corrected for the ROI).

statistical comparison (OT vs PLA) yielded no significant result. ROI analyses of amygdala or right fusiform gyrus did not lead to significant results in any of the contrasts either.

Specific Aspects of Social Processing. Besides these effects on general processing of social stimuli, we also investigated more specific aspects of social processing.

Matching task (contrasts 'negative faces $>$ non-social', 'positive faces $>$ non-social', 'negative scenes $>$ non-social', and 'positive scenes $>$ non-social'): Analyzing each social condition of the matching task separately resulted in brain activation patterns comparable to that of the combined contrast 'social $>$ non-social' with strong bilateral amygdala and fusiform gyrus activation ( $p<0.05$ FWE-corrected).

Genotype effects in the single conditions were also comparable to those of the combined contrast. A- carriers showed stronger activation in the left fusiform gyrus in each

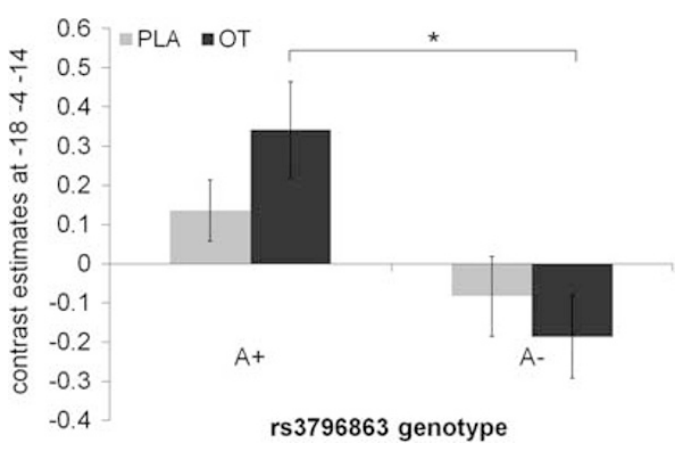

Figure 4 Mean contrast estimates \pm SEM for the left amygdala peak voxel of the contrast 'direct>averted' (both substance conditions combined) in the gaze processing paradigm. * Indicates significant differences ( $p<0.05$ FWE-corrected for the ROI).

of the social task conditions (compared with the non-social forms) combining both substance conditions (OT + PLA, $p<0.05$ FWE-corrected for the ROI). Although under PLA, there were either no or only small clusters higher activated in the A- group, this allele effect was much stronger under OT ( $p<0.05$ FWE-corrected for the ROI) (Supplementary Table S2). None of the statistical comparisons using the difference contrasts OT vs PLA survived FWE-correction procedures for multiple testing. ROI analyses of right fusiform gyrus or amygdala also did not detect any differences between genotypes in the single conditions contrasts.

Gaze processing task (contrasts 'direct>averted' and 'averted $>$ direct'): In the whole sample, analyses of both substance conditions combined (OT + PLA) revealed only a single cluster in the right dorsolateral prefrontal cortex ( $k=19,(56,34,14), t=5.6, p=0.012$ FWE-corrected), which showed higher activation during processing of direct gaze compared with averted gaze. Whereas the opposite contrast 'averted $>$ direct' led to a stronger activation in the left premotor cortex $(k=98,(-24,-8,56), t=6.27$, $p=0.001$ FWE-corrected).

Comparing genotypes during processing of direct $v s$ averted gaze did not result in different activation patterns in fusiform gyri in both substance conditions combined (OT + PLA), nor separately (OT and PLA) or in their comparison (OT vs PLA). With regard to amygdala activation, we found a significantly higher activation of the amygdala bilaterally in $\mathrm{A}+$ carriers compared with $\mathrm{A}-$ for both substance conditions combined (OT + PLA, left cluster: $k=38,(-18$, $-4,-14), t=4.26, p=0.002$ FWE-corrected for the ROI; right cluster: $k=10,(20,0,-12), t=3.37, p=0.019$ FWE-corrected for the ROI). Analyzing both substance conditions separately, this difference could not be observed under PLA, but only in the left amygdala under OT $(k=37$, $(-18,-2,-18), t=3.49, p=0.014$ FWE-corrected for the ROI) (Figure 4). However, the statistical comparison of OT $v s$ PLA yielded no significant results.

\section{DISCUSSION}

Our findings provide evidence that $r s 3796863$ impacts social processing on a very basic level. Participants homozygote for the ASD risk allele (A-, genotype CC) showed a locally specific greater activation of left fusiform gyrus during 
social processing in two independent paradigms. As this effect was observable during processing of emotional and neutral facial stimuli as well as social scenes, it seems not to be restricted to emotion or facial perception but to be a general mechanism taking effect during the processing of social stimuli. Previous studies on face processing in ASD often reported hypoactivation of fusiform gyri, so we originally also had expected the higher risk group $(\mathrm{A}-)$ to show hypoactivation of fusiform gyri (Jemel et al, 2006; Schultz, 2005). However, there is evidence that fusiform activation increases with longer fixation of the eye region in patients, first degree relatives and also in healthy individuals (Dalton et al, 2005, 2007; Morris et al, 2007). Given that usually patients and relatives exhibit shorter eye fixation times, which lead to hypoactivation of fusiform areas, one could hypothesize that they would revert to normal or even hyperactivation if they exhibited 'normal' scanpaths. In our study all subjects were healthy individuals and hence probably do not show any serious alterations of visual scanning paths like ASD patients do. Accordingly, the increased neural activity in fusiform gyrus together with longer RT indicates a less efficient basal visual social processing system in the higher risk group. It seems possible that in case of ASD that this less efficient system cannot be compensated through increased activation and may result in a breakdown of the system leading to deficits in social processing. Of course, there are other possible explanations for the increased fusiform activation. Eg one could argue that the increased activation derives from reduced habituation to social stimuli in homozygotic riskallele carriers. In fact it could be shown that in ASD patients amygdala habituation to facial stimuli is reduced (Kleinhans et al, 2009). However, this was not found for fusiform gyrus where patients and controls did not differ regarding habituation (Kleinhans et al, 2009). Therefore, this still remains unclear. Another point to be discussed is that differences in behavior (RT) could account for differences in fMRI data. But as we only found behavioral differences in one of the two paradigms and moreover stimulus presentation times were independent from subjects' responses we don't believe behavioral differences to explain our fMRI results.

So far, we can only speculate how the homozygotic occurance of the risk allele may lead to stronger fusiform activation. As the $\mathrm{C}$ allele was found to be associated with lower CD38 expression in lymphoblastoid cells in ASD patients (Lerer et al, 2010) and lower CD38 expression to result in reduced OT secretion (Jin et al, 2007) it is not clear how reduced OT secretion results in higher fusiform activation. So far, in humans and primates there is no evidence that the fusiform gyrus could be a potential direct target of OT in terms of existing OT receptors (Loup et al, 1991; Schorscher-Petcu et al, 2009). Therefore, it could be speculated that OT probably affects fusiform activation via so far unknown mechanisms, perhaps via interacting with other neurotransmitter systems like eg the dopamine system. The dopamine system is supposed to have a crucial role for rewarding social affiliative behaviors (which are in turn regulated by $\mathrm{OT}$ ) and could thereby contribute to ASD's social disinterest and deficits (Baskerville and Douglas, 2010; Skuse and Gallagher, 2009). This idea is compatible with a model of the pathophysiology of autism
(Schultz, 2005) stating that alterations in the amygdalafusiform system are the critical neurobiological mechanism underlying ASD's deficits in face perception. A congenital abnormality of the amygdala and the closely connected dopaminergic reward system would then lead to a failure to orient to salient social stimuli such as faces, thereby preventing the development of facial expertize, which is represented in fusiform functioning. As the amygdala seems to be a potential target of OT (Baumgartner et al, 2008; Domes et al, 2007a; Kirsch et al, 2005; Petrovic et al, 2008), this reduced salience signaling of amygdala to social stimuli could be caused by alterations in the OT system.

However, as we only investigated healthy individuals, it is not possible to transfer that model (Schultz, 2005) on a oneto-one basis. Especially, our results do not provide any evidence for genotype-dependent alterations in amygdala activation during basal social processing. Nevertheless, it could be speculated that risk allele carriers exhibit aberrant development of their amygdala-fusiform system during childhood and adolescence and that these alterations finally result in a less efficient system in adulthood.

Regarding interactions within the OT system it seems likely that in the initial state reduced OT secretion in the higher risk group leads to an upregulation of OT receptor density and sensitivity resulting in an hypersensitivity to OT as it has already been described for other neurotransmitter systems for a long time (Burt et al, 1977). Experimentally elevated OT levels could then induce an increased activation of the OT system especially in homozygotic risk-allele carriers and finally lead to even stronger fusiform activation and improved behavioral performance as reported in the present study. However, one should keep in mind that acceleration of RT was not specific for social processing, but was also found in processing of non-social forms, which indicates a general facilitation of visual processing instead of a specific social facilitation effect under elevated OT levels. None of the previous studies on therapeutic effects of OT in ASD patients investigated whether improved performances were specific for social processing or if they also occur during non-social cognitive tasks (Andari et al, 2010; Guastella et al, 2010; Hollander et al, 2007). Therefore, this question requires further investigation.

With respect to the amygdala we did not find any differences in activation during basal social processing depending on allelic makeup. We also were not able to confirm former results showing increased amygdala activation under direct gaze compared with averted gaze in the whole group (Kawashima et al, 1999). However, recent evidence suggests that the amygdala response to gaze direction is dependent on different factors like the viewer's anxiety level and besides, these effects strongly differ with respect to different subnuclei of the amygdala (Ewbank et al, 2010; Hoffman et al, 2007; Straube et al, 2010). Therefore, our result of increased amygdala activation in the $\mathrm{A}+$ group under OT adds to evidence for an additional OT level and genotype-dependent reactivity of the amygdala to direct $v s$ averted gaze.

There are some shortcomings of our study. Firstly, we did not implement a face localizer task to identify individual localization of fusiform face area. Accordingly, we cannot infer if the effect is specific for face processing or rather an effect of processing of complex visual stimuli. Secondly, one 
has to keep in mind the multiple comparisons problem, which usually occurs in fMRI studies and is as yet unresolved. We performed lots of tests and our study's design would have allowed to perform numerous other tests because of the high number of possible combinations of regressors. But in order to avoid further complexity, we decided to concentrate on those contrasts described above. Lastly, we had only two homozygous risk-allele carriers in our sample and thus cannot conclude anything about genedose-effects, which might be present. All these points strongly emphasize the need for further exploration of our results in a replication dataset.

Altogether our study identified alterations in left fusiform gyrus activation as a potential brain mechanism through which the risk variant of $r s 3796863$ could contribute to ASD's social deficits. We also found OT to enhance fusiform activation differences between allele groups and to improve $\mathrm{RT}$ only in the higher risk group, thereby clearly supporting the idea of treating ASD patients with OT. However, one has to keep in mind that the risk variant of $r s 3796863(\mathrm{~A}-)$ is not rare at all with $\sim 50 \%$ occurrence in the Caucasian population. Accordingly, the reported imaging phenotype is also very common and cannot explain the profound social deficits found in ASD patients. Therefore, we rather believe it to be one among lots of different mechanisms involved in deviant social processing in ASD. Correspondingly, any potential therapeutic effect of OT on social functioning in ASD is only partially mediated by the reported imaging phenotype or the underlying genotype. Apart from these constraints, very recently it has been shown that retinoids can be used to increase CD38 expression (Riebold et al, 2011) implicating a direct method to influence the CD38 system and related social cognitive deficits in autism (Ebstein et al, 2011). Finally, in this context, our findings indicate that one should take into account the individual genetic makeup when evaluating treatment effects.

\section{ACKNOWLEDGEMENTS}

We thank Dagmar Gass and André Spachmann for their support during data acquisition. Moreover, we thank Katrin Morgen and Luanna Dixson for proof reading the manuscript. This study was supported by grants from the German Research Foundation (DFG) to PK (KI 576/10-1) and MR (RE 1692/4-1).

\section{DISCLOSURE}

The authors declare no conflict of interest.

\section{REFERENCES}

Andari E, Duhamel JR, Zalla T, Herbrecht E, Leboyer M, Sirigu A (2010). Promoting social behavior with oxytocin in highfunctioning autism spectrum disorders. Proc Natl Acad Sci USA 107: 4389-4394.

Baskerville TA, Douglas AJ (2010). Dopamine and oxytocin interactions underlying behaviors: potential contributions to behavioral disorders. CNS Neurosci Ther 16: e92-123.

Baumgartner T, Heinrichs M, Vonlanthen A, Fischbacher U, Fehr E (2008). Oxytocin shapes the neural circuitry of trust and trust adaptation in humans. Neuron 58: 639-650.
Bradley MM, Lang PJ (1994). Measuring emotion: the selfassessment manikin and the semantic differential. J Behav Ther Exp Psychiatry 25: 49-59.

Burt DR, Creese I, Snyder SH (1977). Antischizophrenic drugs: chronic treatment elevates dopamine receptor binding in brain. Science 196: 326-328.

Dalton KM, Nacewicz BM, Alexander AL, Davidson RJ (2007). Gaze-fixation, brain activation, and amygdala volume in unaffected siblings of individuals with autism. Biol Psychiatry 61: $512-520$.

Dalton KM, Nacewicz BM, Johnstone T, Schaefer HS, Gernsbacher MA, Goldsmith HH et al (2005). Gaze fixation and the neural circuitry of face processing in autism. Nat Neurosci 8: 519-526.

De Dreu CK, Greer LL, Handgraaf MJ, Shalvi S, Van Kleef GA, Baas $M$ et al (2010). The neuropeptide oxytocin regulates parochial altruism in intergroup conflict among humans. Science 328: 1408-1411.

De Dreu CK, Greer LL, Van Kleef GA, Shalvi S, Handgraaf MJ (2011). Oxytocin promotes human ethnocentrism. Proc Natl Acad Sci USA 108: 1262-1266.

Ditzen B, Schaer M, Gabriel B, Bodenmann G, Ehlert U, Heinrichs $M$ (2009). Intranasal oxytocin increases positive communication and reduces cortisol levels during couple conflict. Biol Psychiatry 65: 728-731.

Domes G, Heinrichs M, Glascher J, Buchel C, Braus DF, Herpertz SC (2007a). Oxytocin attenuates amygdala responses to emotional faces regardless of valence. Biol Psychiatry 62: 1187-1190.

Domes G, Heinrichs M, Michel A, Berger C, Herpertz SC (2007b). Oxytocin improves 'mind-reading' in humans. Biol Psychiatry 61: 731-733.

Ebstein RP, Mankuta D, Yirmiya N, Malavasi F (2011). Are retinoids potential therapeutic agents in disorders of Social cognition including autism? FEBS Lett 585: 1529-1536.

Ekman P, Friesen W (1976). Pictures of Facial Affect. Consulting Psychologists Press: Palo Alto.

Ewbank MP, Fox E, Calder AJ (2010). The interaction between gaze and facial expression in the amygdala and extended amygdala is modulated by anxiety. Front Hum Neurosci 4: 56.

Franke GH (2002). Die Symptom-Checkliste von Derogatis (SCL-90-R) - Deutsche Version. Beltz Test GmbH: Göttingen.

Freese JL, Amaral DG (2005). The organization of projections from the amygdala to visual cortical areas TE and V1 in the macaque monkey. J Comp Neurol 486: 295-317.

Freese JL, Amaral DG (2006). Synaptic organization of projections from the amygdala to visual cortical areas TE and V1 in the macaque monkey. J Comp Neurol 496: 655-667.

Gregory SG, Connelly JJ, Towers AJ, Johnson J, Biscocho D, Markunas CA et al (2009). Genomic and epigenetic evidence for oxytocin receptor deficiency in autism. BMC Med 7: 62.

Guastella AJ, Einfeld SL, Gray KM, Rinehart NJ, Tonge BJ, Lambert TJ et al (2010). Intranasal oxytocin improves emotion recognition for youth with autism spectrum disorders. Biol Psychiatry 67: 692-694.

Guastella AJ, Howard AL, Dadds MR, Mitchell P, Carson DS (2009). A randomized controlled trial of intranasal oxytocin as an adjunct to exposure therapy for social anxiety disorder. Psychoneuroendocrinology 34: 917-923.

Guastella AJ, Mitchell PB, Dadds MR (2008). Oxytocin increases gaze to the eye region of human faces. Biol Psychiatry 63: 3-5.

Hariri AR, Mattay VS, Tessitore A, Fera F, Smith WG, Weinberger DR (2002a). Dextroamphetamine modulates the response of the human amygdala. Neuropsychopharmacology 27: 1036-1040.

Hariri AR, Mattay VS, Tessitore A, Fera F, Weinberger DR (2003). Neocortical modulation of the amygdala response to fearful stimuli. Biol Psychiatry 53: 494-501.

Hariri AR, Tessitore A, Mattay VS, Fera F, Weinberger DR (2002b). The amygdala response to emotional stimuli: a comparison of faces and scenes. Neuroimage 17: 317-323. 
Haxby JV, Hoffman EA, Gobbini MI (2000). The distributed human neural system for face perception. Trends Cogn Sci 4: 223-233.

Haxby JV, Hoffman EA, Gobbini MI (2002). Human neural systems for face recognition and social communication. Biol Psychiatry 51: 59-67.

Heinrichs M, Baumgartner T, Kirschbaum C, Ehlert U (2003). Social support and oxytocin interact to suppress cortisol and subjective responses to psychosocial stress. Biol Psychiatry 54: 1389-1398.

Higashida H, Lopatina O, Yoshihara T, Pichugina YA, Soumarokov AA, Munesue $\mathrm{T}$ et al (2010). Oxytocin signal and social behaviour: comparison among adult and infant oxytocin, oxytocin receptor and CD38 gene knockout mice. J Neuroendocrinol 22: 373-379.

Hoffman KL, Gothard KM, Schmid MC, Logothetis NK (2007). Facial-expression and gaze-selective responses in the monkey amygdala. Curr Biol 17: 766-772.

Hollander E, Bartz J, Chaplin W, Phillips A, Sumner J, Soorya L et al (2007). Oxytocin increases retention of social cognition in autism. Biol Psychiatry 61: 498-503.

Huber D, Veinante P, Stoop R (2005). Vasopressin and oxytocin excite distinct neuronal populations in the central amygdala. Science 308: 245-248.

Itier RJ, Batty M (2009). Neural bases of eye and gaze processing: the core of social cognition. Neurosci Biobehav Rev 33: 843-863.

Jacob S, Brune CW, Carter CS, Leventhal BL, Lord C, Cook Jr EH (2007). Association of the oxytocin receptor gene (OXTR) in Caucasian children and adolescents with autism. Neurosci Lett 417: 6-9.

Jemel B, Mottron L, Dawson M (2006). Impaired face processing in autism: fact or artifact? J Autism Dev Disord 36: 91-106.

Jin D, Liu HX, Hirai H, Torashima T, Nagai T, Lopatina $\mathrm{O}$ et al (2007). CD38 is critical for social behaviour by regulating oxytocin secretion. Nature 446: 41-45.

Kanwisher N, Yovel G (2006). The fusiform face area: a cortical region specialized for the perception of faces. Philos Trans $R$ Soc Lond B Biol Sci 361: 2109-2128.

Kawashima R, Sugiura M, Kato T, Nakamura A, Hatano K, Ito K et al (1999). The human amygdala plays an important role in gaze monitoring. A PET study. Brain 122(Part 4): 779-783.

Kirsch P, Esslinger C, Chen Q, Mier D, Lis S, Siddhanti S et al (2005). Oxytocin modulates neural circuitry for social cognition and fear in humans. J Neurosci 25: 11489-11493.

Kleinhans NM, Johnson LC, Richards T, Mahurin R, Greenson J, Dawson $G$ et al (2009). Reduced neural habituation in the amygdala and social impairments in autism spectrum disorders. Am J Psychiatry 166: 467-475.

Kosfeld M, Heinrichs M, Zak PJ, Fischbacher U, Fehr E (2005). Oxytocin increases trust in humans. Nature 435: 673-676.

Lang PJ, Bradley MM, Cuthbert BN (2008). International Affective Picture System (IAPS): Affective Ratings of Pictures and Instruction Manual. Technical Report A-8. University of Florida, Gainesville, FL.

Laux L, Glanzmann P, Schaffner P, Spielberger CD (1981). State-Trait-Angstinventar (STAI). Beltz Test GmbH: Göttingen.

Lee HJ, Macbeth AH, Pagani JH, Young III WS (2009). Oxytocin: the great facilitator of life. Prog Neurobiol 88: 127-151.

Lerer E, Levi S, Israel S, Yaari M, Nemanov L, Mankuta D et al (2010). Low CD38 expression in lymphoblastoid cells and haplotypes are both associated with autism in a family-based study. Autism Res 3: 293-302.
Lerer E, Levi S, Salomon S, Darvasi A, Yirmiya N, Ebstein RP (2008). Association between the oxytocin receptor (OXTR) gene and autism: relationship to Vineland Adaptive Behavior Scales and cognition. Mol Psychiatry 13: 980-988.

Loup F, Tribollet E, Dubois-Dauphin M, Dreifuss JJ (1991). Localization of high-affinity binding sites for oxytocin and vasopressin in the human brain. An autoradiographic study. Brain Res 555: 220-232.

Meyer-Lindenberg A, Domes G, Kirsch P, Heinrichs M (2011). Oxytocin and vasopressin in the human brain: social neuropeptides for translational medicine. Nat Rev Neurosci 12: 524-538.

Meyer-Lindenberg A, Weinberger DR (2006). Intermediate phenotypes and genetic mechanisms of psychiatric disorders. Nat Rev Neurosci 7: 818-827.

Morris JP, Pelphrey KA, McCarthy G (2007). Controlled scanpath variation alters fusiform face activation. Soc Cogn Affect Neurosci 2: 31-38.

Munesue T, Yokoyama S, Nakamura K, Anitha A, Yamada K, Hayashi K et al (2010). Two genetic variants of CD38 in subjects with autism spectrum disorder and controls. Neurosci Res 67: 181-191.

Petrovic P, Kalisch R, Singer T, Dolan RJ (2008). Oxytocin attenuates affective evaluations of conditioned faces and amygdala activity. J Neurosci 28: 6607-6615.

Riebold M, Mankuta D, Lerer E, Israel S, Zhong S, Nemanov L et al (2011). All-trans retinoic acid upregulates reduced CD38 transcription in lymphoblastoid cell lines from autism spectrum disorder. Mol Med 17: 799-806.

Schorscher-Petcu A, Dupre A, Tribollet E (2009). Distribution of vasopressin and oxytocin binding sites in the brain and upper spinal cord of the common marmoset. Neurosci Lett 461: 217-222.

Schultz RT (2005). Developmental deficits in social perception in autism: the role of the amygdala and fusiform face area. Int J Dev Neurosci 23: 125-141.

Schwenkmezger P, Hodapp V, Spielberger CD (1992). State-TraitÄrgerausdrucks-Inventar (STAXI). Verlag Hans Huber: Bern.

Skuse DH, Gallagher L (2009). Dopaminergic-neuropeptide interactions in the social brain. Trends Cogn Sci 13: 27-35.

Steyer R, Schwenkmezger P, Notz P, Eid M (1997). Der Mehrdimensionale Befindlichkeitsfragebogen (MDBF). Hogrefe: Göttingen.

Straube T, Langohr B, Schmidt S, Mentzel HJ, Miltner WH (2010). Increased amygdala activation to averted $v s$ direct gaze in humans is independent of valence of facial expression. Neuroimage 49: 2680-2686.

Tost H, Kolachana B, Hakimi S, Lemaitre H, Verchinski BA, Mattay VS et al (2010). A common allele in the oxytocin receptor gene (OXTR) impacts prosocial temperament and human hypothalamic-limbic structure and function. Proc Natl Acad Sci USA 107: 13936-13941.

Verhoeven JS, De Cock P, Lagae L, Sunaert S (2010). Neuroimaging of autism. Neuroradiology 52: 3-14.

Wermter AK, Kamp-Becker I, Hesse P, Schulte-Korne G, Strauch $\mathrm{K}$, Remschmidt $\mathrm{H}$ (2010). Evidence for the involvement of genetic variation in the oxytocin receptor gene (OXTR) in the etiology of autistic disorders on high-functioning level. $\mathrm{Am} \mathrm{J}$ Med Genet B Neuropsychiatr Genet 153B: 629-639.

Wu S, Jia M, Ruan Y, Liu J, Guo Y, Shuang M et al (2005). Positive association of the oxytocin receptor gene (OXTR) with autism in the Chinese Han population. Biol Psychiatry 58: 74-77.

Yrigollen CM, Han SS, Kochetkova A, Babitz T, Chang JT, Volkmar FR et al (2008). Genes controlling affiliative behavior as candidate genes for autism. Biol Psychiatry 63: 911-916.

Supplementary Information accompanies the paper on the Neuropsychopharmacology website (http://www.nature.com/npp) 